\title{
Materialität und Narration im Hörbuch
}

Überlegungen am Beispiel deutscher und französischer Hörbuchfassungen von Tausendundeine Nacht

Matérialité et narration dans le livre audio : réflexions à partir de versions audio allemandes et françaises des Mille et Une Nuits

Materiality and Narration in the Audiobook: The Example of German and French Audio Versions of Thousand and One Nights

\section{Kerstin HAUSBEI}

\section{OpenEdition}

Journals

Édition électronique

URL : http://journals.openedition.org/ceg/3662

DOI : 10.4000/ceg.3662

ISSN : 2605-8359

Éditeur

Presses Universitaires de Provence

Édition imprimée

Date de publication : 15 novembre 2018

Pagination : 107-122

ISBN : 979-10-320-0183-7

ISSN : 0751-4239

\section{Référence électronique}

Kerstin HAUSBEI, « Materialität und Narration im Hörbuch », Cahiers d'Études Germaniques [Online], 75 2018, Online erschienen am: 25 April 2020, abgerufen am 26 November 2020. URL : http:// journals.openedition.org/ceg/3662 ; DOI : https://doi.org/10.4000/ceg.3662 


\section{Materialität und Narration im Hörbuch Überlegungen am Beispiel deutscher und französischer Hörbuchfassungen von Tausendundeine Nacht}

Kerstin HAUSBEI

Université Sorbonne Nouvelle / CEREG (EA 4223)

Hörbücher sind in den letzten beiden Jahrzehnten - im deutschsprachigen Raum mehr als im französischsprachigen - zu einer relativ weit verbreiteten Form der Literaturrezeption geworden. ${ }^{1}$ Um die Konsequenz des Medienwechsels für die Rezeption von narrativen Texten zu erfassen, soll im Folgenden die Relation zwischen Materialität und Narration ins Auge gefasst werden, wobei unter Materialität ein Phänomen der Wahrnehmung verstanden wird, bei dem die Aufmerksamkeit am phänomenalen So-Sein der Dinge „hängenbleibt“ und diese aus ihrer denotativen Verweisstruktur herauslöst. ${ }^{2}$ Statt ,auf etwas“ (hier die Diegese) zeigen im Wahrnehmungsmodus der Materialität die Zeichenträger (hier beispielsweise Stimme, Sprache und Trägermedium) ,sich selbst: Sie werden sinnlich wahrgenommen und dabei als anziehend, abstoßend usw. empfunden, wobei gleichzeitig eine „Fülle von Assoziationen, Wirkungen, Gefühlen, Erinnerungen, Imaginationen und Gedanken“ freigesetzt wird, die ihrerseits in emergente Bedeutungsvielfalt umschlägt. ${ }^{3}$ Wenn es nun bei der Rezeption von Hörbüchern - wie bei jeder ästhetischen Kommunikation - zu einem Oszillieren zwischen Referentialität und Materialität kommt und dem Rezipienten unabhängig von der Produzentenintention prinzipiell alles zum Zeichen werden kann, so erzeugt Materialität bei der narrativen Sinnkonstitution Zusatzinformationen in Form von Selbstreferentialität und ästhetischer Bedeutungsvielfalt. Es ist vor diesem Hintergrund davon auszugehen, dass der narrative Sinn durch Dispositive, Techniken und Materialitäten erst

1. In Deutschland stieg der Umsatz im Zeitraum von 1995 bis 2010 von 2 Mio. Euro auf 300 Mio. Euro. 2010 lag der Anteil am Buchmarkt mit 25000 Titeln (jährlicher Zuwachs: 800 Titel) bei 3,4\%. In Frankreich waren 2010 nur 5000 Titel verfügbar (jährlicher Zuwachs: 110 Titel). Vgl. Gert Ueding, Lemma „Hörbuch“, in ders. (Hrsg.), Historisches Wörterbuch der Rhetorik, Band 10, Berlin, De Gruyter, 2012, S. 365.

2. Vgl. Sabine Schouten, Lemma „Materialität“, in Erika Fischer-Lichte, Doris Kolesch, Matthias Warstat (Hrsg.), Metzler Lexikon Theatertheorie, Stuttgart/ Weimar, Metzler, 2005, S. 194-196. $\mathrm{Zu}$ philosophischen Gesichtspunkten vgl. Dieter Mersch, Was sich zeigt. Materialität, Präsenz, Ereignis, München, Fink, 2000.

3. Vgl. Schouten, „Materialität“, S. 195. 
bedingt wird. ${ }^{4}$ Das Hörbuch lässt sich deshalb auch weder allein über das Speichermedium definieren ${ }^{5}$, noch als reine „Verstimmlichung von Literatur“ 6 , bei der dem Sprecher die „Funktion eines Digital-Analog-Wandlers“7 zukommt. Fasst man den Begriff des Materials im allgemeinsten Sinn als Stoff, der zur Weiterverarbeitung vorgesehen ist, so stellt das Hörbuch die Zusammenführung dreier Materialien dar: das zuvor in schriftlicher Form existierende sprachliche und narrative Material, das stimmliche oder - weiter gefasst - akustische Material und ein Trägermedium. So entsteht „ein akustischer Text“8, dem, wie Jürg Häusermann fordert, der Status einer „Neuedition“ zugebilligt werden sollte. ${ }^{9}$ Allerdings ist davon auszugehen, dass die neue materielle Verfasstheit auch die narrative Struktur des Textes grundlegend verändert. So weist Johannes E. Lehmann darauf hin, dass Materialitätseffekte im Hörbuch schon allein daraus resultieren, dass sich in der Rezeption zwei widersprüchliche Zeitlichkeiten - die durch das Medium Sprache und das Speichermedium dem Hörer aufgenötigte prozessuale Linearität und die der Inszenierung zugrundeliegende globalisierende Interpretation des Gesamttextes - so überlagern, dass sie in ein Spannungsverhältnis zueinander treten. ${ }^{10}$ Durch die Charakteristika der Sprechstimmen, den Sprechrhythmus und prosodische Mittel machen die „,schrift'-gestützte[n] ,Sprech“-Weisen der ,sekundären Oralität““"11 insgesamt aus dem ,Sprach'-Kunstwerk ein ,Sprech'-Kunstwerk ${ }^{12}$, das zu der Textgrundlage in eine spezifische Relation tritt und bestimmte Sinnpotentiale materialisiert, die der Hörer nicht verändern kann. Definiert man mit Franz K. Stanzel die Narrativität im Wesentlichen durch das Kriterium der Mittelbarkeit, so fügt das Hörbuch dem narrativen Ausgangstext mit Sprecher und technischem Medium zwei neue Vermittlungsinstanzen hinzu, zu denen aber in den meisten Fällen noch weitere kommen. Der materiellen Verfasstheit der Erzählung im Hörbuch kommt insofern ein ähnlicher Status zu wie der Diskursebene der Erzählung: Die Diegese tritt dem Publikum in einer spezifischen Form wie durch einen

4. Vgl. Elias Kreuzmair, „Hans Ulrich Gumbrechts Begriff der Präsenz und die Literatur“, in Helikon. A Multidisciplinary Online Journal 2, 2012, S. 233-247.

5. So aber Gert Ueding: „Hörbücher sind Sprachtonträger, die sich unterschiedlicher technischer Aufzeichnungs- und Wiedergabegeräte bedienen“ („Hörbuch“, S. 365).

6. Stephanie Bung, Jenny Schrödl, „Vorwort“, in dies. (Hrsg.), Phänomen Hörbuch. Interdisziplinäre Perspektiven und medialer Wandel, Bielefeld, transcript Verlag, 2016, S. 10.

7. Heinz Hiebler, „Problemfeld ,Hörbuch: Das Hörbuch in der medienorientierten Literaturwissenschaft", in Natalie Binczek, Cornelia Epping-Jäger (Hrsg.), Das Hörbuch. Praktiken audioliteralen Schreibens und Verstehens, München, Fink, 2014, S. 102.

8. Bung, Schrödl, „Vorwort“, S. 9.

9. Vgl. Jürg Häusermann, „Das Hörbuch zwischen öffentlicher Lesung und privater Rezeption“, in Ursula Rautenberg (Hrsg.), ,Hörbuch“ - Stimme und Inszenierung, S. 64.

10. Vgl. Johannes E. Lehmann, „Literatur lesen, Literatur hören. Versuch einer Unterscheidung“, Text und Kritik 96: Literatur und Hörbuch, 2012, Gasthrsg. Natalie Binczek, Cornelia Epping-Jäger, S. 4 und S. 9.

11. Hiebler, „Problemfeld ,Hörbuch“ “, S. 102.

12. Vgl. Tilla Schnickmann, „Vom Sprach- zum Sprechkunstwerk. Die Stimme im Hörbuch: Literaturverlust oder Sinnlichkeitsgewinn?“, in Ursula Rautenberg (Hrsg.), Das Hörbuch - Stimme und Inszenierung, Wiesbaden, Otto Harrassowitz, 2007, S. 21-54. 
Filter entgegen, der publikumslenkende Funktionen wie die des Kommentars, der Perspektivierung und Distanzregulierung übernimmt. Materialitätseffekte entstehen vor allem, wenn entweder gegen das Prinzip der „Gestaltganzheit“, also der „stilistischen Einheit als Bedingung für Textualität“" ${ }^{13}$, verstoßen wird oder ein akustisches Element in Bezug auf das Sinnverstehen des Hörers, das mitgeprägt ist von seinem (auch gattungsorientierten) Erwartungshorizont, spontan nicht semantisierbar ist. Durch die Bindung an den Erwartungshorizont ist Materialität natürlich individuell und historisch variabel. Das Oszillieren der Aufmerksamkeit des Zuhörers kann aber auch durch inszenatorische Techniken bewusst erzeugt und gelenkt werden und trägt dann nicht nur zum Sinnverstehen der ,Geschichte' bei, sondern vehikuliert auch eine Vorstellung davon, was Erzählen ist und welche Funktion das jeweilige Hörbuch gegenüber dem vorgängigen Erzähltext einnimmt.

Diesem Phänomen werden wir am Beispiel von deutschen und französischen Hörbuchvertonungen des Erzählzyklus Tausendundeine Nacht nachgehen. Der Zyklus bietet sich an, weil er durch seine bewegte Entstehungs- und Überlieferungsgeschichte ${ }^{14} \mathrm{zu}$ einem „offenen Werk“ geworden ist, was sich in einem breiten Formenspektrum akustischer „Neueditionen“ niederschlägt. Als eine der prominentesten Erzählungen über das Erzählen ist in ihm aber auch Selbstbezüglichkeit schon angelegt. Bekanntlich erzählt in der wohl zweitausend Jahre alten Rahmengeschichte die belesene und kluge Schahrasad ${ }^{15}$ in einer von ihr inszenierten doppelten Kommunikationssituation scheinbar ihrer Schwester Dinarasad, eigentlich aber ihrem Ehemann, dem Sultan Schahriyar, Nacht für Nacht Geschichten, um ihren Tod (und den der Jungfrauen des Landes) hinauszuzögern und letztlich zu verhindern. Notwendig ist diese Maßnahme, weil der Sultan es sich nach dem Ehebruch seiner ersten Frau zur Regel gemacht hat, jede Nacht eine Frau zu entjungfern und dann zu töten. Erzählen wird also in der Kommunikationssituation der Diegese als Dispositiv - als Problemlösungsoperator - eingeführt, wodurch die Aufmerksamkeit des Rezipienten auf die Narrativität selbst gelenkt wird. So hängt das Verzögern des Todesurteils davon ab, dass Geschichten ihrem Wesen nach geschlossene Handlungs- und Sinneinheiten sind, das Einsetzen und Abbrechen des Erzählens aber einem davon unabhängigen äußeren Faktor, dem Lauf der Sonne, unterworfen wird. Verlängert werden die Sinneinheiten dadurch, dass die Einzelgeschichten wiederum das Erzählen mehrerer Geschichten enthalten, also selbst in Zyklen organisiert sind, wobei auch die Situation der Rahmengeschichte - Todesurteil durch eine höhere Instanz und Auslösung des

13. Ulla Fix, „Nichtsprachliches als Textfaktor: Medialität, Materialität, Lokalität“, in Zeitschrift für Germanistische Linguistik 36/ 3, 2008, S. 343-353.

14. Vgl. für einen raschen Überblick das Nachwort von Claudia Ott zu ihrer Übersetzung des von Muhsin Mahdi edierten Textes (Tausendundeine Nacht, München, Beck, 2011) oder Ulrich Marzolph, „Die Erzählungen aus Tausendundeine Nacht als Monument transnationaler Erzählkunst“, in Märchen 2, 2008, S. 75-93. Für eine fundierte Darstellung Aboubakr Chraïbi, Les Mille et Une Nuits. Histoire du texte et classification des contes, Paris, L'Harmattan, 2008.

15. Alle Figurennamen in der Orthographie Claudia Otts. 
Verurteilten durch das Erzählen von Geschichten - in den Binnengeschichten gespiegelt erscheint und der Tauschwert der Geschichten hier wie dort von ihrer Erstaunlichkeit und deren Steigerung abhängt. Dieses mehrdimensionale Verschachtelungs- und Vernetzungsprinzip geht wohl erst auf die älteste erhaltene arabische Fassung, die so genannte „Galland-Handschrift“, zurück ${ }^{16}$, in der die ursprünglichen Geschichten mit direkt belehrendem Inhalt weitgehend verändert und ersetzt wurden zugunsten eines Kompositionsprinzips, das die Notwendigkeit der Erzeugung von Hörermotivation ebenso thematisch werden lässt wie die anthropologische Komponente des autobiographischen Erzählens als Ich-Konstruktion (Todorov spricht in diesem Zusammenhang vom „homme-récit“" ${ }^{17}$ ). Diese Komposition führt zu einer komplexen Überlagerung von Kommunikationssituationen, Erzählinstanzen und Sprechhandlungen, so etwa, wenn der Erzähler Schahrasad zum Sultan sagen lässt: „Es ist mir zu Ohren gekommen, dass der Küchenchef dem Kaiser von China erzählte, dass der junge Mann sagte [...].“ Diese Dialogizität, nach Bachtin ein Gattungsmerkmal von Erzähltexten ${ }^{18}$, stellt für das Hörbuch eine Herausforderung dar.

Neben der Überlagerung von Erzählsituationen eignet dem Erzählzyklus ein ebenfalls in der Rahmengeschichte angelegter parataktischer Zug. Dieses Prinzip der Reihung übte die Wirkung eines „Magneten“ 19 aus und ist dafür verantwortlich, dass dem anonym tradierten „Buch ohne Autor“ ${ }^{20}$ in mehreren Schüben und verschiedenen Kombinationen neues Erzählmaterial aus diversen historischen und kulturellen Kontexten einverleibt werden konnte, weshalb es sich „nur bedingt [...] um ein Werk mit eindeutig definiertem Inhalt“ ${ }^{21}$ handelt. Das Geschichtenrepertoire wurde auch in der europäischen Rezeptionsphase zu Beginn des 18. Jahrhunderts noch wesentlich erweitert (unter anderem durch Hinzufügung der heute bekanntesten Geschichten wie „Sindbad, der Seefahrer“, „Ali Baba und die vierzig Räuber“ und „Aladin und die Wunderlampe“). Wie Claudia Ott betont, ist „der ,abendländische“ Beitrag zur Überlieferung“ - zu dem neben neuen Geschichten auch eine Orientierung am europäischen Publikumsgeschmack gehört - „heute Teil auch des arabischen Verständnisses des Textes“ und zieht daraus den Schluss, dass - nach dem Transfer von der indischen in die vor-islamisch persische und der darauffolgenden Islamisierung des Textes in der arabischen Kultur - Tausendundeine Nacht nun eine „westöstliche Geschichte“ geworden sei. ${ }^{22}$

Die anonyme Tradierung des Zyklus führte auch $\mathrm{zu}$ sprachlicher und stilistischer Heterogenität. ${ }^{23}$ Die Sprachregister umfassen ein breites Spektrum

16. Vgl. Chraïbi, Les Mille et Une Nuits, S. $60 f$.

17. Vgl. Tzvetan Todorov, Poétique de la prose (choix) suivi de Nouvelles recherches sur le récit, Paris, Seuil, 1980.

18. Vgl. Michael Bachtin, Die Ästhetik des Wortes, Frankfurt a.M., Suhrkamp, 1979.

19. Vgl. Ott, „Nachwort“, S. 644.

20. Ibid., S. 645.

21. Marzolph, „Die Erzählungen aus Tausendundeine Nacht“, S. 79.

22. Ott, „Nachwort“, S. 647.

23. Vgl. für die folgenden Erklärungen Ott, „Nachwort“. 
vom Dialekt bis zum klassischen Hocharabisch und auf stilistischer Ebene stehen neben der Erzählprosa auch Gedichte, Koran-Zitate und Sprichwörter, sowie die Reimprosa (arabisch Sadsch), eine Abfolge kurzer gereimter Sätze oder Satzteile. Erhebliche Variationen gibt es auch zwischen den einzelnen arabischen Manuskripten, die sich in den aus ihnen hervorgegangenen Übersetzungen niederschlagen. So betont Claudia Ott die „Nähe zur Mündlichkeit“ der von ihr übersetzten „Galland-Handschrift“ in Abgrenzung von dem „literarisierten, der Schriftform angepassten Stil“ des Enno Littmann übersetzten Drucks „Calcutta II“. ${ }^{24}$ Entgegen populärwissenschaftlichen Behauptungen handelt es sich bei Tausendundeine Nacht aber nicht um verschriftlichte, ursprünglich orale Literatur. ${ }^{25}$ Die Hörbücher können insofern auch nicht als Rückführung in eine frühere Textphase verstanden werden. Wohl aber hat der Erzählzyklus zum Repertoire der Hakawati, der professionellen Erzähler auf öffentlichen Plätzen, gehört und ist in diesem Rahmen sekundär vermündlicht worden. Die untersuchten Hörbuchfassungen setzen an sehr verschiedenen Stellen dieses „offenen Werkes“ an.

\section{Lesungen mit einem Sprecher ohne Musik und Geräusche}

Jede Stimme erzeugt bei ihrem Einsetzen einen Materialitätseffekt: „Sie ist Leib und überraschende Nacktheit zugleich“ ${ }^{26}$, „Objekt des Begehrens - oder des Abscheus“27. Die Sprecherstimme ist dem entsprechend, zumal bei Lesungen mit einem Sprecher ohne Musik und Geräusche, von großer Bedeutung für die Aufmerksamkeitsgewinnung des Hörers, tritt aber in der Wahrnehmung wohl recht schnell hinter das Sinnverstehen zurück: „Sie ist schwer zu fixieren, weil die Stimme unablässig durch das überformt wird, was sie sagt: Eine Bedeutung schiebt sich vor ihre Gegenwart, verkleidet und verdeckt sie.“ ${ }^{28}$ Im Hörbuch erfolgt diese Verschmelzung von Stimme und Narration vor allem dann, wenn Stimme und Verstimmlichung dem Erwartungshorizont des Hörers, der jeweils aktuellen Hörmode und auch der Hörästhetik des Mediums und der Textgattung entsprechen. In unserem Korpus fällt auf, dass viele Stimmen sich aus unterschiedlichen Gründen dieser Verschmelzung widersetzen.

Dazu gehört die Lesung des Kinder- und Jugendbuchautors Dirk Walbrecker, der 2010 für den Kuebler Hörbuchverlag seine eigene Textfassung von „Ali Baba und die vierzig Räuber“ einsprach, einer Geschichte der europäischen Tradierung also, die ihren festen Platz im Repertoire der Märchenliteratur hat. Entgegen dem gattungsbezogenen Erwartungshorizont, geprägt von professionellen Erzählstimmen, deren prosodische Effekte gezielt dazu eingesetzt werden, kleinen

24. Ibid., S. 650.

25. Vgl. Chraïbi, Les Mille et Une Nuits, S. 17-19.

26. Dieter Mersch, „Präsenz und Ethizität der Stimme“, in Doris Kolesch, Sibylle Krämer (Hrsg.), Stimme. Annäherungen an ein Phänomen, Frankfurt a.M., Suhrkamp, 2006, S. 211.

27. Roland Barthes, zitiert nach Mersch, „Präsenz“, S. 212.

28. Ibid. 
Kindern den Mitvollzug der Handlung zu ermöglichen, verfügt Walbreckers Stimme nicht über die klangliche Breite und die Variationsmöglichkeiten eines ausgebildeten Sprechers. Sein leichter regionaler Akzent aus dem Nordwesten Deutschlands (deutlich hörbar am „a“-Laut) verstößt nicht nur gegen die Gattungserwartung, sondern ist auch weder in Bezug auf die Herkunft des Textes noch auf den Handlungsort semantisierbar. Die Stimme erzeugt so einen Materialitätseffekt, kann allerdings assoziativ verbunden werden mit der Stimmästhetik der Autorenlesung, die auf „nackte Stimmen“ als Authentizitätseffekt setzt, um die Persönlichkeit des Autors als Zusatzinformation mit seinem Text in Bezug zu setzen. ${ }^{29}$ Der Materialitätseffekt verrät hier also die Bemühung, den Text im Literaturkanon neu zu situieren, worauf auch die zweieinhalbstündige Dauer hinweist, die sich deutlich vom Bearbeitungsformat in der Märchentradition abhebt. Der Cover-Text („Walbreckers Klassiker für Kids“) bestätigt diese Vermutung. Ob Walbrecker sich als Autor gegenüber einem so bekannten Text etablieren kann, darf allerdings als zweifelhaft gelten.

Der Radiolesung von Gisela Trowe ${ }^{30}$ aus dem Jahr 1975 dürfte bei dem Versuch einer Neueinordnung des Zyklus in den Literaturkanon ungleich mehr Erfolg beschieden sein. Dafür ist paradoxerweise zunächst die weniger perfektionierte Tontechnik verantwortlich, die durch Störgeräusche (Rauschen in den Sprechpausen, metallener Klang der Stimme, Zischlaute, Verzerrung mancher Wortendungen) die Wahrnehmung des Hörers auf die technische Vermittlung lenkt. Wird die Sprecherin so eindeutig als Abwesende wahrgenommen, verleihen ihr die ebenfalls deutlich hörbaren Schluck- und Atemgeräusche gleichzeitig eine Körperlichkeit, die sie als Sprechinstanz deutlich markiert und zwischen den Hörer und den Text stellt. Weitere Materialitätseffekte lassen die vorzeitige schrifttextliche Natur der Textquelle hervortreten. Dazu gehört sowohl die satzzeichenbetonte Stimmführung als auch die erzählerbetonte Gestaltung der Dialoge, die sich rhythmisch und melodisch nicht von der textlichen Umgebung abheben, sowie die bemüht arabische Aussprache einiger Wörter (etwa des Wortes „Scheich“, zunächst mit deutschem ei-Laut ausgesprochen, dann ab dem zweiten Vorkommen mit ej-Laut). Auch stilistische und klangliche Eigenschaften der Textfassung von Enno Littmann erzeugen Materialitätseffekte, die nunmehr die Literarität der Textquelle betonen. Neben dem hochsprachlichen, leicht antiquierten Stil ${ }^{31}$ sorgen vor allem die deutlich hörbaren Sadsch-Reime ${ }^{32}$ für eine Selbstbezüglichkeit der Sprache als Klang. Schließlich tragen auch die

29. Vgl. zur Ästhetik der Autorenlesung Reinhart Meyer-Kalkus, „Literatur für Stimme und Ohr“, in Brigitte Felderer (Hrsg.), Phonorama. Eine Kulturgeschichte der Stimme als Medium, Berlin, Matthes \& Seitz, 2004, S. 173-186.

30. Die Erzählungen aus Tausendundeins Nächten, Lesung von Gisela Trowe aus dem Jahr 1975, Reihe swr2 Lesung, swr-edition.

31. Durch die Verwendung von „ward“ statt „wurde“, hinzugefügten e-Laut bei Endungen auf „d“, ausgefallene Wortwahl usw.

32. So gleich zu Beginn: „Es wird berichtet - Allah aber ist Allwisser seiner verborgenen Dinge und Allherrscher und allgeehrt und allgnädig und allgütig und allbarmherzig - in den Erzählungen aus alter Zeit und aus der Völker Vergangenheit, dass in früheren Tagen, die weit 
gesprochenen Titel der Geschichten dazu bei, die schriftliche Textquelle als Vermittlungsinstanz für den Hörer präsent zu halten, und das trifft auch auf den deutlich hörbaren Schnitt zwischen den einzelnen Geschichten zu, der zwar die bearbeitende Instanz hervortreten lässt, aber gerade durch den von ihr vollzogenen Bruch die Aufmerksamkeit des Hörers auf den (hier verletzten) Werkcharakter der vorgängigen Textquelle zieht. In deutlicher Absetzung von der in den 1970er Jahren noch stark dominanten geschichtenorientierten Rezeption in der Märchentradition (auch die Auswahl der Geschichten verletzt übrigens diesen Erwartungshorizont und schafft einen Materialitätseffekt) wird also hier nicht nur die Mittelbarkeit zum Hauptmerkmal des Erzählens, sondern führen alle Materialitätseffekte den Hörer dazu, das Hörbuch als sekundäres Medium im Dienste eines Werkes zu verstehen, dessen Literarität das hervorstechendste Merkmal ist. Dabei wird allerdings die komplexe Verschachtelungsstruktur ebenso unterschlagen wie die stilistische Offenheit des „Werks ohne Autor“.

In der wesentlich geschichtenorientierteren Lesung Katharina Thalbachs ${ }^{33}$ entsteht ein dauerhafter Materialitätseffekt durch die ausgeprägte Eigencharakteristik ihrer Stimme. Ihr raues, tiefes, leicht nasales Timbre fällt nicht nur aus dem Stimmprofil der meisten Hörbuchsprecher heraus, sondern erzeugt auch einen starken Wiedererkennungseffekt, durch den hinter der Stimme die Person zur Erscheinung gebracht wird. Als Fremdtextsprecherin verkörpert sich in ihr die Erzählinstanz, und so entsteht für den Hörer der Eindruck einer direkten Kommunikationssituation, wozu auch die Halllosigkeit der Studioaufnahme beiträgt, durch die die Erzählstimme scheinbar raumlos in intimen Bezug zu ihm tritt. Die durch die Rahmengeschichte gesetzte diegetische Erzählsituation wird am Anfang des Hörbuchs auf ein einfaches (und nicht erklärtes) „Herr“ reduziert, wodurch die Figur Schahrasad zwar formal präsent ist, das Hörbuch sich aber ganz auf das Erzählen der ausgewählten Binnengeschichte („Aladin und die Wunderlampe“) konzentriert. Katharina Thalbach spielt dementsprechend auch nicht die Erzählerin Schahrasad, für deren Profil ihre Stimme auch denkbar unpassend wäre, sondern leiht diese einer auktorialen Erzählinstanz. Sie liest inhaltsbezogen in expressiver Lektüre, wobei die stimmlichen Effekte eine publikumslenkende Kommentarfunktion übernehmen (also beispielsweise Reprobation, Bedauern, Erstaunen in Bezug auf das Erzählte ausdrücken), wichtige Momente der Handlung durch den Sprechrhythmus aus dem Erzählfluss herausheben und so die Geschichte aus der Gesamtkenntnis des Textes heraus perspektivieren. Im Gegensatz zu Schahrasad werden die Figuren der Diegese bei Dialogen momentweise gespielt, also stimmlich charakterisiert und in ihrer jeweiligen Sprechhandlung und Gemütsverfassung gezeigt. Jedoch behält Katharina Thalbach auch in diesen Momenten den regelmäßigen Sprechrhythmus bei, der auf die Schriftlichkeit der Textquelle verweist, und

in entschwundene Zeitalter ragen, ein König der Sasaniden im Inselreiche von Indien und China lebte“ [zitiert nach dem Hörbuch; Hervorhebung $\mathrm{KH}$ ].

33. Aladin und die Wunderlampe, Übersetzung von Gustav Weill, Lesung von Katharina Thalbach, Freiburg, Audiobuchverlag, 2015. 
wechselt zwischen engagiertem und distanziertem Lesen hin und her, wodurch eher der Eindruck einer mimetischen Skizze denn einer Verwandlung in die Figur entsteht. Deutlich wird so die Figurendarstellung der Erzählerinstanz zu- und untergeordnet. Erst am Ende der über fünfstündigen Lesung wird das bisher Erzählte Schahrasad als Erzählinstanz zugewiesen. Die wesentlichen Elemente der Rahmengeschichte (Geschichtenerzählen als Lebensrettung, Prinzipien der Reihung und der Steigerung) werden nun situativ eingeführt und von den Figuren auch explizit erörtert. Stimmlich wird die Erzählweise der Binnengeschichte auf der höheren Erzählebene exakt reproduziert, wodurch in der Art einer Metalepse die Binnengeschichte vom Hörer weggerückt und die bisherige Erzählsituation als Trompe-l'œil entlarvt wird. Besonders auffällig, weil unüblich, sind die Temposteigerung, die deutlich erhöhte Sprechintensität und der melodische Hochschluss, mit denen Katharina Thalbach das Hörbuch bei der letzten Replik des Sultans („Lass uns jetzt sehen, was du Neues zu erzählen hast“) stimmlich regelrecht ,abbrechen“ lässt. Gerade diese Forderung löst das Hörbuch ja auch nicht mehr ein. Der Erzählpakt wird dadurch aufgekündigt. Was eine Geschichte ist, wird dem Hörer im Entzug bewusst, wobei diese ästhetische Erfahrung exakt der des Sultans korrespondiert. Der Hörer wird so in Form einer ästhetischen Erfahrung zumindest ansatzweise mit der komplexen Selbstbezüglichkeit des Gesamtwerks konfrontiert.

\section{Lesung durch einen Sprecher mit Musik und Geräuschen}

Ebenfalls geprägt durch eine charakteristische Stimme ist die Radiolesung von Fernandel aus dem Jahr 1958. ${ }^{34}$ Sie umfasst die „europäischen“ Geschichten „Ali Baba et les quarante voleurs", „Aladin et la lampe magique“ und „Sindbad, le marin“. Fernandel, der Ali Baba 1954 schon im Kino verkörpert hatte ${ }^{35}$, verstimmlicht die Geschichten mit einem starken Marseiller Akzent. Das erzeugt einen dauerhaften Materialitätseffekt einerseits in Bezug auf den medialen Erwartungshorizont (die am Theater orientierte, akzentfreie Sprechästhetik des Hörfunks), andererseits in Bezug auf die Gestaltganzheit, denn Fernandel singt die Eingangslieder (das Titellied des „Ali Baba“-Films und ein „Sindbad“-Lied) akzentfrei. Die Geschichten werden durch den Akzent vermündlicht (wobei die Satzmelodie allerdings durch Satzzeichenbetontheit deutlich auf die Schriftquelle verweist) und assoziativ in den französischen Kulturraum versetzt, wozu auch die Musikkomposition in Chansontradition und der Ortsname „Hispan“ beitragen, der am Anfang fällt. All diese Elemente sind auf ein kindliches Publikum abgestimmt, dem die Geschichten aus dem fremden Kulturraum - stark bearbeitet und zusammengedrängt auf das für Kindergeschichten übliche zehnminütige Format - angenähert werden. Anders als bei Walbrecker evoziert der Akzent

\footnotetext{
34. Les contes des Mille et Une Nuits, Lesung mit Musik und Geräuschen von Fernandel aus dem Jahr 1958, Ulysse, 2010.

35. Ali Baba et les quarante voleurs von Jacques Becker.
} 
hier jedoch einen mit der Erzählung kohärenten mediterranen Kulturraum. Für den Kenner der Textgeschichte kann die Zusatzinformation „französisch“ auch den Akt „kultureller Einverleibung“ zeigen, den die Bearbeitung von Galland ja durchaus darstellt.

Der kunstvoll eingesetzte Akzent und die Bekanntheit der Stimme erzeugen eine starke, selbstbezügliche Präsenz des Sprechers als Vermittlungsinstanz. Die Stimme wird in dieser Funktion unterstützt von situativ nicht eingebundenen Geräuschen, die isoliert, in einer jeweils ähnlichen zeitlichen Ausdehnung der Erwähnung desselben Elements (leicht nach-, manchmal auch vorgeordnet) unterlegt werden: Erwähnung der Esel > Schreien der Esel; schnelles Hufgetrappel und Glockengeläut > Erwähnung des Hinwegs ohne Ladung usw. So findet hier kein akustisches Überblenden auf die diegetische Ebene im Sinne einer Dramatisierung oder eines fingierten 0 -Ton-Features $\operatorname{statt}^{36}$, sondern die Geräusche sind auf der Ebene des Erzählers zu verorten, fungieren als akustische Zeichen und sind dadurch semantisch und formal redundant in ihrem Verhältnis zum Text. Wohl aber enthält ihre Künstlichkeit in ihrem So-Sein eine Zusatzinformation, da diese eine leicht ironische Distanzierung erzeugt (beispielsweise, wenn das Öffnen und Schließen des Eingangs zur Höhle wie ein quietschender Kurbelmechanismus klingt), die als Fiktionalitätssignal verstanden werden kann. Auch stimmlich hält Fernandel stets eine vermittelnde Distanz zur Diegese. Nur aus der Erzählerwarte nähert er sich den Figurenperspektiven (vor allem deren emotiven Zuständen) an. So werden die Figuren auch bei direkter Rede nicht gespielt, wohl aber kann der Sprecher ihren inneren Gemütszustand kommentierend zum Ausdruck bringen: Beispielsweise spricht Fernandel die Formel „Sésame, ouvre-toi“, wenn Ali Baba sie zum ersten Mal benutzt, leise und ein wenig schüchtern, als müsse er seinen ganzen Mut zusammennehmen, während das einleitende Verb „crier“ ein lautes Rufen nahelegt. Hier entsteht in der Diskrepanz der Informationen ein Materialitätseffekt, der als Mehrfachperspektivierung im Sinne von Bachtins Dialogizität verstanden werden kann. Erzählen erscheint bei Fernandel gleichgewichtet als Vermittlung einer „Geschichte“ (Chatman, Ricoeur) ${ }^{37}$ und als „Vermittlung“ einer Geschichte (Genette, Stanzel) ${ }^{38}$, wobei aber auf die Literarität und Komplexität der Textvorlage völlig verzichtet wird.

36. Ein solcher Effekt wird beispielsweise bei der Hörspielbearbeitung von Karlheinz Koinegg in der Inszenierung von Angeli Backhausen (1001 Nacht. Scheherazad und der Brunnen der Geschichten, Westdeutscher Rundfunk/ Hörverlag 2014) intendiert, wenn im Hintergrund der Erzählung Geräusche des Marktes oder der Arbeit von Handwerkern eingespielt werden. Hier werden auch dramatisierte Szenen eingespielt, in denen durch Hall und Geräusche fiktive Räume konstruiert werden, in denen die Figuren durch ihre Distanz zum Mikrophon genau situiert werden. Mit diesen Elementen kontrastiert die Stimme der Erzählerin, die „raumlos“, also ohne jeden Hall eingespielt wird.

37. Vgl. Seymour Chatman, Coming to terms: the rhetoric of narrative in fiction and films, Ithaca, Cornell UP, 1990, und Paul Ricoeur, Temps et récit, Band 1, Paris, Seuil, 1983.

38. Vgl. Gérard Genette, Figures III, Paris, Seuil, 1972, und Franz K. Stanzel, Theorie des Erzählens, Göttingen, Vandenhoeck \& Ruprecht, 1978. 


\section{Vermündlichung}

Auch die Stimmen von Jihad Darwiche ${ }^{39}$ und von Saddek Kebir ${ }^{40}$ erzeugen Materialitätseffekte durch Akzente: ein nicht leicht zuzuordnender Akzent im Französischen bei dem Libanesen Darwiche, ein französischer Akzent im Deutschen bei dem Algerier Kebir. Beide Erzähler stellen sich in die arabische Tradition der Hakawati und stellen so den Aspekt der mündlichen Vermittlung einer Erzählung an ein Publikum in den Vordergrund, dem gegebenenfalls auch gewisse Elemente des Textes erläutert werden müssen. Dabei setzen sie aber völlig unterschiedliche Akzente.

Die Lesung von Jihad Darwiche beruht wesentlich auf der klangatmosphärisch erzeugten Teilhabe an einer kulturlandschaftlich verankerten Erzählpraxis. Entsprechend beginnt das Hörbuch mit der musikalischen Erzeugung eines Klangraums. Mit Mikrophon und Halleffekten verstärkt werden zunächst hölzerne Klopfgeräusche und metallene Klänge, wie sie beim Schütteln eines Donnerblechs im Theater entstehen, erzeugt, die in einem Resonanzraum nachklingen. In diesem akustisch erzeugten Raum erklingt nun eine Flöte, die die lang angehaltenen Töne deutlich als Luftstrom kennzeichnet, manchmal leicht moduliert und teils wie unkontrolliert in Obertöne abgleiten lässt. Noch bevor die Sprechstimme einsetzt, wird so ein warmer atmosphärischer Raum erzeugt, der meditativ wirkt und assoziativ mit Naturbildern von karger Weite und einem mediterran-orientalischen Kulturraum (etwa der Vorstellung von Hirten in der Wüste) verbunden werden kann. Erst nach einer vollen Minute ertönt die Stimme Darwiches gleichzeitig mit diesem Klangraum, allerdings mit einer vollkommen anderen Tonästhetik, da hier auf jeden Halleffekt verzichtet wird, die Stimme also raumlos klingt. Die Narration wird hier in die Musik hineingestellt und das setzt sich auch nach dem Verschwinden der Instrumente (nach weiteren 30 Sekunden) fort. Dazu trägt die überraschende Klanglichkeit, vor allem aber die eigenwillige Satzrhythmik und -melodie der Stimme Darwiches bei, die eine starke, von der Narration zum Teil unabhängige, Eigendynamik entwickelt, die einerseits zu genauem Hinhören zwingt, andererseits aber auch der Sprache eine starke Musikalität ,beifügt', die ihr hier durch die Stimmführung quasi von außen erst zukommt. Dazu gehört ein fast als arabischer AchLaut ausgesprochenes $\mathrm{R}$ ebenso wie die Dehnung von Vokalen entgegen den phonetischen Regeln des Französischen. Gefolgt wird diese Dehnung von einer Beschleunigung des Sprechrhythmus bis zum Ende des Satzes, wobei einige Silben fast verschluckt werden können. Einige immer wiederkehrende Wörter - vor allem die thematischen Signalwörter „homme“ (mit extrem kurzem Vokal und Verweilen auf dem m), „femme“ (lang gedehnt), „il a dit“ (mit lang gedehntem i) und „marchand“ - werden wie musikalische Leitmotive eingesetzt,

39. Les Mille et Une Nuits, dans les pas de Shéhérazade, Lesung von Jihad Darwiche aus dem Jahr 2005. Komposition, Gitarre, Flöte, Trommel und Gesang von Abaji, unter der künstlerischen Leitung von Pascal Dubois, Valence, oui'dire éditions, 2010.

40. Erotische Märchen aus 1001 Nacht, erzählt von Saddek Kebir, swr 2 Lesung, swr edition, 1994. 
die die Kreisstruktur unterstreichen, die den Zyklus durch immer wiederkehrende Erzählmotive prägt. Diese der (europäisch-westlichen) Linearität der Narration entgegengesetzte (arabisch-orientalische) Kreisstruktur wird von Darwiche durch die immer wiederkehrende Sequenz „Dehnung/ Beschleunigung/ Pause“ auch in die einzelnen kurzen Sätze transponiert. Er verbindet diese musikalische Struktur mit narrativen Akzentuierungen, verzichtet aber völlig auf das Spielen von Figuren oder auf die sprecherische Gestaltung des narrativen Ebenenwechsels. Er bewegt sich so im Rahmen eines Sprechkunstwerks, das durch Mündlichkeit (einfacher Satzbau, einfaches Vokabular, parataktisches Nebeneinanderstellen der Geschichten) geprägt ist, an kunstvolle arabisch-orientalische Traditionen des professionellen mündlichen Erzählens anknüpft und den Hörer zur atmosphärischen Teilhabe daran einlädt.

Demgegenüber wird bei Saddek Kebir die Kultur-,Vermittlung“ als kommunikative Handlung mit Materialitätseffekten belegt. Dafür ist einerseits von Bedeutung, dass es sich um den Mitschnitt einer Aufführung vor Publikum handelt, dessen Reaktionen (vor allem Lachen) immer wieder hörbar sind, andererseits dass Kebir produzierend (spontan) formuliert, die aktuelle Kommunikationshandlung also gegenüber der schriftsprachlichen Quelle profiliert. Der für ein erzählendes Hörbuch ohnehin ungewöhnliche Dokumentationscharakter tritt in ein paradoxales Verhältnis zur Tonästhetik der Stimm-Aufnahme, die den Performer nicht in die Schallakustik des Aufführungsraums integriert, sondern ähnlich wie bei einer Studioaufnahme (wohl durch ein am Kopf befestigtes Mikrophon und die Nachbearbeitung der Tonspur im Studio) die Erzählerstimme raumlos nah an den Hörer heranholt. Sie ermöglicht eine (im Aufführungsraum normalerweise unmögliche) breite Modulationspalette bis hin zum Flüstern, wie sie heute auch im Theater praktiziert wird. Medientechnisch paradoxal wirkt das ebenfalls unübliche spontane Sprechen, das sich in Zögern und sachlich falschen Formulierungen materialisiert, die in einer zusätzlichen Sprechhandlung korrigiert werden müssen, was vor allem auf der Bildebene Materialitätseffekte erzeugt durch die Ersetzung eines vom Hörer schon vorgestellten Bildes (zum Beispiel: „Er setzte sich auf eine Bank. Also auf einen Stein“). Für den Hörer entsteht so eine doppelte Kommunikationssituation, in der er gleichzeitig Adressat und unbeteiligter Zeuge einer spontanen mündlichen Erzählung ist. Paradoxal ist auch die stark profilierte Sprecherpersönlichkeit gestaltet, da Kebir sprecherische Virtuosität mit sprachlicher Fragilität, ja „Nacktheit“, verbindet, die sich in zahlreichen Sprachfehlern (Konjugations-, Syntax-, Genusfehler usw.) und in falschen Silbenbetonungen und Satzmelodien zeigt und im Hörbuch ausgesprochen ungewöhnlich ist. Zur Kongruenz kommen Sprechen und Sprache allein, wenn Kebir Arabisch spricht, wobei die Kommunikation dann aber mit hoher Wahrscheinlichkeit daran scheitert, dass der Hörer ihn nicht versteht. Das Hörbuch beginnt bezeichnenderweise mit einer solchen Passage in arabischer Sprache, wobei der Sprecher durch Betonungen und rhythmische Effekte die ästhetische Qualität des zitierten Originaltextes herausstellt. Erst bei der nun folgenden Übertragung der Passage ins Deutsche wird dem Hörer der Sinn 
zugänglich, hier nun verliert er aber durch die Vermündlichung des Erzählstils und die fehlerhafte Sprache die Literarität der Schriftquelle: „Es gab ,mal' in alten Zeiten einen Sultan. Der Sultan ,heißt' Schahryiar. Jede Nacht,wollt'er' eine Jungfrau haben, mit der er ,schlieft“ [...]." Es ,zeigt sich so die Vermittlungsleistung des Hakawati, der seinem Publikum in mündlicher Kommunikation didaktisch einen Schrifttext erschließt. Er überbrückt die Distanz, die das Publikum vom Text trennt, macht diese aber auch als kulturellen ,Zwischen'-Raum spürbar, in dem er seine eigene Position zwischen dem Hörer und der erzählten Geschichte geschickt moduliert. So unterstreicht er (beispielsweise durch die arabische Grußformel „salam aleikum“ oder die Kommentierung von Figurenverhalten als typisch muslimisch) immer wieder den sprachlichen und kulturellen Abstand der Diegese von seinem Publikum und inszeniert sich auch selbst in diesem kulturellen Abstand, beispielsweise wenn er - scheinbar reflexartig - bei der Erwähnung des Wortes „Sultan“ die Formel „Es gibt keinen Sultan außer Allah“ nachschiebt. Gleichzeitig werden alle diese Elemente (unter anderem durch exzessive Wiederholung) ironisch gebrochen, erscheinen also als kulturelle Konstruktionen. Auf der anderen Seite nähert Kebir sich dem Publikum und schafft eine gemeinsame (zeitliche und ontologische) Distanz zur Diegese durch ironisch-komische Erläuterungen, die oft mit einem „Verstehen Sie“ eingeleitet werden, durch stimmliche Mittel der Perspektivierung (vor allem die übertriebene Darstellung von Gefühlen der tyrannischen Figur des Sultans), sowie durch die Kollision zwischen der Verwendung von konkretem, aktuellem Vokabular aus der Alltagssprache (etwa dem englischen Lehnwort „Baby“) in Verbindung mit der fantastischen Sphäre der erzählten Welt (hier ein Geist). Kebir verringert auch den Abstand des Publikums zur arabischen Sprache, einerseits durch die Verwendung von für ihn transparenten Formeln wie dem erwähnten „salam aleikum“ (wohingegen unüblichere Ausdrücke wie „Iffrit“ übersetzt werden), andererseits aber auch durch die beharrliche Wiederholung von Sadsch-Formeln am Anfang der Geschichten, die jedes Mal wieder übertragen werden, aber beim Hörer einen deutlichen Wiedererkennungseffekt provozieren, ihn also lustvoll an die fremde Sprache heranführen. Erzählen zeigt sich so bei Kebir kulturell als orientalische Praxis, anthropologisch als Selbstverständigung und zwischenmenschliche Verständigung und gesellschaftlich als kulturelle Vermittlungsarbeit, die eine - auch politische - Funktion in der Zielgesellschaft erfüllt. Der Hörer ist dabei gleichzeitig Zielpublikum ,und“ Zeuge der interkulturellen Kommunikation, dem sich der Sinn des Erzählens durch Materialitätseffekte auf vielerlei Weise erschließt. 


\section{Lesung mit mehreren Sprechern}

Die 2005 entstandene Lesung der Übersetzung von Claudia 0tt ${ }^{41}$ positioniert sich im Covertext explizit als Neuedition: „Das arabische Original - erstmals in deutscher Übersetzung“. Durch professionelle Sprecherstimmen ohne starke Eigencharakteristik, einen gemessenen Sprechrhythmus und vermittelnden Erzählstil kommt sie dem Erwartungshorizont des Hörers entgegen. Materialität wird in ihr allein durch die Aufkündigung der Gestaltganzheit erzeugt und dient der Profilierung des Originaltextes. So wird an der Textoberfläche die stilistische Heterogenität der Textvorlage umgesetzt, wodurch die dynamische Entwicklung der Handlung immer wieder von nicht narrativen Textelementen unterbrochen wird. Auch die Gestaltganzheit der übergeordneten Erzählerstimme ist aufgegeben (Einsatz von mehreren Sprechern), ohne dass jedoch eine für den Hörer ebenfalls spontan sinnvolle Einheit „Stimme/ Figur“ an ihre Stelle gesetzt würde (Alle Sprecher leihen ihre Stimme Schahrasad). Der Genderkontrast kann vom Hörer auf den Wechsel der Erzählebenen bezogen werden: Während Heikko Deutschmann als einziger männlicher Sprecher die Rahmengeschichte einschließlich der in ihr enthaltenen Dialoge und Geschichten erzählt, wird immer dann eine weibliche Sprecherin eingeführt, wenn Schahrasad zur Erzählerin einer Binnengeschichte wird. Wie der Sultan von der Sonne, wird der Hörer durch die männliche Stimme aus der jeweiligen Geschichte gerissen und entwickelt so durch Frustration ein Bewusstsein dafür, was eine Geschichte ist. Die durch den Stimmwechsel exponierte fast wörtliche Wiederholung der immer gleichen Elemente in den Einschüben der Rahmengeschichte (Sonnenaufgang und Einbruch der Nacht, Lob der begonnenen Geschichte, Ankündigung der Steigerung und Bitte um die Vollendung der begonnenen Geschichte), die durch die fast exakte Reproduktion der prosodischen Gestaltung noch unterstrichen wird, führt dem Hörer die Überlagerung der dynamischen Handlungsstruktur der Binnengeschichten mit der Kreisstruktur der Rahmenerzählung, deren Handlung sich über lange Strecken nicht dynamisch entwickelt, deutlich vor Augen. Irritierender wirkt der insgesamt siebenmalige Sprecherinnenwechsel für die ,Erzählerin“ Schahrasad, gerade weil er ,nicht‘ zu einer starken Variation der Stimm- und Sprechästhetik führt, was bei einer sechsundzwanzigstündigen Lesung immerhin für legitime Abwechslung gesorgt hätte. Erst spät versteht der Hörer, dass jeder Sprecherinnenwechsel den Beginn eines neuen Geschichtenzyklus markiert. Die durch Materialitätseffekte erzeugten Zusatzinformationen bringen dem Hörer die komplexe Diskursebene des Zyklus nahe. Gerade dadurch geraten sie hier aber zu reinen Zeichen.

41. Tausendundeine Nacht, mit Heikko Deutschmann, Eva Mattes, Marlen Diekhoff, Charlotte Schwab, Elisabeth Schwarz und Katja Riehmann, Hamburg, Hörbuch Hamburg, 2004. 


\section{Hörspiel}

Mit der komplexen Textstruktur befasst sich auch die Hörspielbearbeitung von Helma Sanders-Brahms, die unter der Regie von Robert Matejka und mit einer Vielzahl von teils sehr prominenten Sprechern, Musik und zahlreichen akustischen Effekten in unserem Korpus eindeutig die aufwendigste Produktion darstellt. ${ }^{42}$ Im Gegensatz zu den anderen Hörbüchern, bei denen die Materialitätseffekte entweder stabil in die Gestaltganzheit mit eingebettet sind oder diese punktuell durchbrechen, könnte man hier fast sagen, dass die Gestaltganzheit daraus entsteht, dass ständig aufs Neue Materialitätseffekte produziert werden, der Hörer also in stetiger Bewegung bleibt. Noch bevor die (im gesprochenen Paratext als „erste Nacht“ eingeführte) Rahmengeschichte einsetzt, wird der Hörer in eine von ihm unabhängige Klangwelt eingeführt, in der sich mehrere Klangebenen durch Mischung überlagern. Die Klänge scheinen hier unabhängig voneinander zu sein, ja sich zu stören, und sind in einem akustischen Raum in Entfernung vom Hörer situiert. Es überlagern sich Klanghölzer, die Laute wie von fließendem Wasser erzeugen, eine Flötenmelodie, eine zweite, anscheinend in arabischer Sprache gesungene Melodie, schließlich auch Trommeln- und Lautenklänge. Das Ganze ergibt einen orientalischen Eindruck, ohne sich zu einem geschlossenen Musikstück zu fügen. Räumlich vor dieser Klangatmosphäre und begleitet im Hintergrund von leise angedeutetem Frauenglucksen ertönt nun eine geflüsterte Männerstimme mit fremdem Akzent: „Ich biete sie euch zum Geschenk. Ganz nackt. Noch jungfräulich. Intakt. Naiv. Den Lüsten und Genüssen meiner Freundinnen und Freunde. Diese arabischen Nächte. Erlebt, erträumt und übertragen von ihrer heimatlichen Erde und übers Meer." Es entstehen so Assoziationen von Erotik, von Geheimnis, von Fremde und (Menschen-) Handel. Nachdem die instrumentalen Klänge sich nun zu einer rhythmisch strukturierteren Musik geordnet haben, beginnt nach dieser eineinhalbminütigen Einleitung eine stimmlich nicht ausgebildete Männerstimme die Erzählung als Lesung aus dem arabischen Original. ${ }^{43}$ Im akustischen Raum befindet sich diese Stimme auf der gleichen Ebene wie die Klangkulisse. Abwechselnd mit dieser Stimme, die insgesamt drei recht lange Passagen auf Arabisch vorträgt, und begleitet von der im Raum situierten Musik setzt nun lauter die raumlose professionell ausgebildete Stimme von Jürgen Hentsch als Erzähler in deutscher Sprache ein. Hat man zunächst den Eindruck, dass es sich um die Übersetzung des arabischen Textes handelt, so wird dieser Eindruck schnell dadurch zunichte gemacht, dass das Original sich offensichtlich in Teilen wiederholt, während die deutsche Stimme narrativ voranschreitet. Entgegen der gerade aufgebauten Erwartung des Hörers wird nun der arabische Text fallengelassen

42. Helma Sanders-Brahms, 1001 Nacht, Regie Robert Matejka, Musik Günter Baby Sommer, Produktion Deutschlandradio Berlin/ RIAS Berlin/ Norddeutscher Rundfunk 1993/ 1994/ 1996, München, Verlag der Autoren/ Der Hörverlag, 2002/ 2003/ 2008.

43. Die Stimme taucht im Sprecherverzeichnis mit der Rollenverteilung nicht auf. Handelt es sich um den Flötenspieler Ali Reza Asgharnia Goorabjeri? 
und die deutsche Erzählerstimme von denotativen Geräuschen begleitet, die eindeutig auf der Erzählerebene angesiedelt sind. Von der originalsprachlichen Textvorlage wird dadurch die Selbstbezüglichkeit auf die erzählerische Vermittlung umgelenkt. Wie bei Fernandel erzeugt die Künstlichkeit der Geräusche eine ironische Kommentarebene, die sich später bis zu einer an Monty Python gemahnenden skurrilen Komik steigert, wenn etwa Schahsaman seiner Frau und ihrem Liebhaber die Köpfe abschlägt und diese wie Bälle durch den Raum dotzen. Der im ästhetischen und assoziativen Bruch vollzogene Materialitätseffekt thematisiert dabei selbstrefentiell Kulturalität, die sich schon im Nebeneinander der Sprachen angedeutet hatte. Konkret tritt dem Hörer hier die west-östliche Ko-Produktion entgegen und sensibilisiert ihn rückblickend auch für die Verwendung westlicher Orient-Klischees und -phantasmen in der Einleitung. Auch diese Ebene wird gebrochen, wenn in dramatisierter Form, verbunden mit einem Sprecherwechsel und der Einführung von Raumakustik die diegetische Ebene sich gegenüber dem Erzähler verselbständigt, die Mittelbarkeit also plötzlich fallengelassen wird zugunsten einer mimetischen Darstellungsart. Interessant sind dabei Übergänge, die den Hörer zwingen, einen gehörten Klang umzudeuten. So etwa, wenn ein Gong als denotativer Klang auf der Erzählerebene eingesetzt wird, aber plötzlich als mimetische Klangkulisse im Raum der Diegese fungiert, wo König Schahryar und sein Wesir miteinander sprechen und auch räumlich durch Hall zu verorten sind. Allerdings werden auch auf der mimetischen Ebene Materialitätseffekte erzeugt, die umgekehrt auf die Mittelbarkeit verweisen. Das geschieht dann, wenn ein Sprecher, während er eine Figur verkörpert, plötzlich eine Figurenreplik vermittelnd spricht, die vom Hörer der Figur zugeordnete Stimme also die Funktion der Erzählerinstanz übernimmt, wodurch Dialogizität entsteht. Auch die stimmlich übertriebene Darstellung von Sprechhandlungen oder Gefühlen kann diese Wirkung haben, indem sie neben dem Wortinhalt, der Figurencharakterisierung und der Sprechhandlung auch einen ironischen Zusatzkommentar zum Ausdruck bringt. Es entsteht auf diese Weise erzählerische Instabilität. In der Folge wird das Dispositiv „Erzählerstimme + mimetisch durch andere Stimmen dargestellte diegetische Ebene (mit einer eigenen Schallakustik und eventuell eigener Klangkulisse)“ gemäß dem Verschachtelungs- und Spiegelungsprinzip der Textvorlage auf der Ebene der Binnengeschichten reproduziert. Und diese ohnehin starke Komplexität wird noch dadurch erhöht, dass in dieser Hörbuchfassung die ,Erzählerin“ Schahrasad streckenweise auch als ,Figur' konzipiert ist, die mit weicher Stimme in einer intimen Atmosphäre spricht, die Kommunikationssituation und Sprechhandlung im Schlafzimmer also darstellt. Der Wechsel der Erzählebenen kann sich deshalb auch bei gleichbleibender Sprecherinnenstimme vollziehen, wenn Schahrasad, ihre Erzählung unterbrechend, sich an ihre direkten Zuhörer (den Sultan und die Schwester) wendet und diese antworten. Dabei wird meist mit stimmlicher Erotik gearbeitet. Ganz anders als in den Fassungen, wo eine Erzählerstimme den Hörer zuverlässig durch eine Geschichte führt, wird die Rezeption des Erzählens hier zu einem Gang durch ein Klanglabyrinth, bei dem hinter jeder Ecke eine neue Überraschung harrt, auf die sich der Hörer einstellen muss. 


\section{Zum Schluss}

Materialität und Narration gehen in den untersuchten Hörbüchern eine vielseitige und spannende Beziehung ein, deren Potentialitäten wohl durch die Besonderheiten des „offenen Werkes“ Tausendundeine Nacht noch verstärkt werden. Die hier untersuchten Hörbücher zeigen aber sowohl, dass Materialität im erzählenden Hörbuch immer selbstbezüglich auf die Narrativität zurückweist und so eine Vorstellung dessen vermittelt, was Erzählen ist, als auch, dass Materialitätseffekte nicht nur individuell wahrgenommen, sondern auch inszenatorisch erzeugt und genutzt werden können. In der so entstehenden ästhetischen Erfahrung des Hörers wird Bedeutung konstituiert, die sich mit der Geschichte verknüpft und diese erst erzeugt. So öffnet sich für Hörbücher, wenn sie sich dem modischen Erwartungshorizont widersetzen, ein kreatives Feld, das die Rezeption von Erzählliteratur bereichert. 